Relations industrielles

Industrial Relations

\title{
Le droit du travail, par Pierre-D. Ollier, Paris, Armand Colin,
} 1972, 591 pp.

\section{Pierre Verge}

Volume 28, numéro 2, 1973

URI : https://id.erudit.org/iderudit/028402ar

DOI : https://doi.org/10.7202/028402ar

Aller au sommaire du numéro

Éditeur(s)

Département des relations industrielles de l'Université Laval

ISSN

0034-379X (imprimé)

1703-8138 (numérique)

Découvrir la revue

Citer ce compte rendu

Verge, P. (1973). Compte rendu de [Le droit du travail, par Pierre-D. Ollier, Paris, Armand Colin, 1972, 591 pp.] Relations industrielles / Industrial Relations, 28(2), 431-432. https://doi.org/10.7202/028402ar

Tous droits réservés @ C Département des relations industrielles de l'Universite Laval, 1973
Ce document est protégé par la loi sur le droit d'auteur. L’utilisation des services d'Érudit (y compris la reproduction) est assujettie à sa politique d'utilisation que vous pouvez consulter en ligne.

https://apropos.erudit.org/fr/usagers/politique-dutilisation/ 


\section{RECENSIONS}

Taux du Syndicalisme au Québec, Québec, Ministère du travail et de la main-d'œuvre, Service de la recherche, 1972, 126 pp.

Voici un ouvrage très ambitieux qui nous est présenté par le ministère du Travail et de la Main-d'oeuvre du Québec, celui de tracer une carte syndicale complète du Québec à partir de données statistiques déjà existantes et plus particulièrement de celles fournies d'une part par Organisation des travailleurs au Canada et complétée, d'autre part, en majeure partie par des publications du Bureau Fédéral de la Statistique et du Bureau de la Statistique du Québec.

Ce travail consiste en une longue succession de tableaux représentant, d'abord des données générales par secteur et par organisation syndicale pour ensuite décomposer ces données par soussecteur, par région et en tenant toujours compte de la répartition par organisation syndicale.

Une telle carte syndicale est intéressante à plus d'un point de vue: d'abord elle nous fournit des indications sur le taux de pénétration du syndicalisme dans les différents secteurs concernés par l'étude, soit le primaire, le secondaire, le tertiaire et le secteur de la construction que les auteurs ont considéré à part à juste titre à cause des fluctuations saisonnières de la maind'oeuvre, et ensuite sur la répartition des travailleurs entre les différentes organisations syndicales par secteur et sous-secteur industriel de même que par région.

Nous ne pouvons en effet parler que d'indications à cause des restrictions que les auteurs apportent à la définition d'employés et de syndiqués et celleci est dépendante de celle-là. En effet, on ne considère pas la main-d'oeuvre totale, incluant les chômeurs, mais uniquement les employés, c'est-à-dire ceux qui, à toute fin pratique, avaient un emploi rémunéré durant la semaine de référence. Et alors les syndiqués deviennent «Les employés qui, au cours de la période de référence détenaient une carte de membre d'un syndicat accrédité ou reconnu d'après le Code du travail (p. 117). D'où on ne saurait accorder une fiabilité démesurée aux résultats sans tenir compte du taux de chômage par secteur et par région. L'incidence est peut-être faible dans certains secteurs, mais elle peut être forte dans d'autres et il en est de même pour les régions. Les auteurs auraient dû en tenir compte puisqu'il s'agissait bien d'établir une carte syndicale, donc de décrire statistiquement la pénétration du syndicalisme. De même la simplification de la recherche dans certains cas par le recours aux estimations provoque chez le lecteur une certaine méfiance.

Même si on peut reprocher à cette étude d'avoir été trop ambitieuse, c'està-dire d'avoir choisi de pénétrer profondément à l'intérieur de chaque soussecteur et ce avec le risque de fournir des données incomplètes ou douteuses plutôt que de privilégier une étude approfondie et fiable au niveau des grands secteurs, il n'en demeure pas moins qu'il s'agit là d'un travail utile à tous ceux qui s'intéressent aux relations professionnelles et au syndicalisme.

\section{Gilles LAFLAMME}

Le droit du travail, par Pierre-D. Ollier, Paris, Armand Colin, 1972, 591 pp.

Synthèse, parfois très classique, mais globalement innovatrice, du droit du travail français. L'ouvrage, indique-ton, s'adresse en premier lieu aux étudiants de licence en droit, mais il vise également à atteindre ceux qui oeuvrent, à divers titres, dans la vie économique, de même que le praticien.

L'introduction se range dans la tradition - historique, exposé des sources, organismes chargés de l'application de ce droit. Une première partie étudie le statut du salarié: protection de ce dernier dans sa subordination à l'employeur, pendant la durée et lors de la ces- 
sation du contrat de travail ; étude aussi du salaire. $\mathrm{Si}$, à cette étape également, l'ouvrage ne s'écarte pas tellement des présentations antérieures du contrat de travail, en particulier en ce qui a trait à sa terminaison et à la créance de salaire, il est en revanche assez percutant dans l'analyse qu'il présente du pouvoir patronal. Cette force lui provient de ce que l'auteur se refuse de s'arrêter aux simples «façades juridiques $\gg$, justifications élégantes de certaines solutions jurisprudentielles, pour démasquer au besoin leur fondement anachronique, l'écart qui les sépare souvent d'une réalité sociale, sobrement mais précisément rendue : "Le pouvoir souverain du chef d'entreprise n'est pas autre chose que le droit du propriétaire sur sa chose. L'intérêt de l'entreprise est dès lors un euphémisme dans la motivation des arrêts... (p. 101) » Au même diapason, mais de façon plus particulière, la présentation du droit disciplinaire, inexistant, en somme, «absorbé qu'il est dans le droit du contrat». La position critique qu'adopte l'auteur conserverait, somme toute, sa valeur en droit canadien: «Dans les rapports de travail, qui sont des rapports entre contractants et des rapports d'inégalité, la proportionnalité de la sanction à la faute est une exigence de justice....» (p. 134).

La place prépondérante, en droit français, du contrat individuel, ne prive pas le lecteur d'une substantielle deuxième partie consacrée à la détermination collective des conditions de travail: droit syndical proprement dit, négociation collective et conflits collectifs. Sous ces titres, l'on retrouve, finement analysé, un ensemble usuel de sujets : le mouvement syndical, la liberté syndicale, le rôle de représentation du syndicat, le droit des conventions collectives, de la grève, un certain constat d'inefficacité des modes de règlement pacifique ...

En ces matières, bien qu'il faille tenir compte du public auquel l'on s'adresse, il deviendrait sans doute utile à l'occasion d'entrer plus à fond dans l'examen de certains aspects fondamentaux de grands systèmes étrangers de façon à permettre plus de recul au lecteur dans l'appréciation du système qui le régit. Constamment, en effet - et c'est là la caractéristique la plus fortement positive de l'ouvrage - l'on retrouve cette juxtaposition de l'analyse juridique à la fois nette et serrée, du rappel du contexte socio-économique et de l'invitation à l'appréciation critique.

La troisième partie, «Participation et contrôle », est l'occasion de l'examen des rapports entre les institutions représentatives typiques (délégués du personnel ou comité d'entreprise, selon le cas) et, depuis la loi de 1968, la section syndicale dans l'entreprise - interrogation, pour l'instant, bien française : «Le syndicat doit-il se cantonner dans un rôle de contestation du pouvoir patronal ou participer à l'exercice de ce pouvoir? C'est tout le problème de la réforme de l'entreprise. (p. 450) Exposé également des modes de participation du salarié aux fruits de l'entreprise: «L'intéressement n'est pas la cogestion. Pourtant, comment ne pas voir que c'est cette participation là qui est décisive pour les travailleurs? (p. 510)

Une quatrième partie, «Droit au travail et emploi de la main-d'oeuvre » représente finalement un envol nouveau du droit du travail lequel se fond ici, d'une façon très réaliste, au droit à l'éducation : droit au meilleur emploi, lié à la formation professionnelle et à l'éducation permanente. «Les fondements du droit au travail mettent ainsi en cause le régime économique et son aptitude à se transformer pour satisfaire les besoins essentiels des hommes. (p. 533)

Finalement il est à la fois très pédagogique et très utile de trouver en fin de chapitre, en plus d'une bonne tranche d'une bibliographie sélective, la reproduction même de textes fondamentaux essentiels.

Le droit du travail du professeur Ollier représente sans doute, pour ces catégories de lecteurs français auxquels il se destine essentiellement, l'un des meilleurs précis actuels en la matière. Il est tributaire, à certains égards, de la récente somme publiée sous la direction du professeur Camerlynck. Le lecteur canadien, pour sa part, est mieux en mesure grâce à lui, de percevoir, malgré le particularisme encore très marqué des droits nationaux, l'émergence lente d'un ensemble de préoccupations communes.

Pierre VERGE 